\title{
Expression of Vimentin and Ki-67 Proteins in Cervical Squamous Cell Carcinoma and their Relationships with Clinicopathological Features
}

\author{
Jian-Qin Yu ${ }^{1}$, Qing Zhou ${ }^{1}$, Yun-Fei Zheng ${ }^{1}$, Ying Bao ${ }^{2 *}$
}

\begin{abstract}
Objectives: To investigate the expression of vimentin and Ki-67 proteins in cervical squamous cell carcinoma (CSCC) and their relationships with patient clinicopathological features. Materials and Methods:Fifty-seven CSCC samples archived in Department of Pathology in the First Affiliated Hospital of Wenzhou Medical University were selected. The expression of vimentin and Ki-67 proteins in CSCC tissue were detected using immunohistochemical SP method, and correlations between them and their relationships with clinicopathological features were analyzed. Results: Among $57 \mathrm{CSCC}$ tissues, there were 43 with positive expression of Vimentin, and the positive rate was $75.4 \%$; there were 57 cases with positive expression of $\mathrm{Ki}-67$, and the positive rate came up to $100.0 \%$. The results of Pearson correlation analysis displayed that the expression of vimentin had a significantly-positive correlation with Ki-67 in CSCC tissue $(r=0.984, c 00.000)$. The expression of both Ki-67 and vimentin was intimately associated with the presence or absence of local invasion and lymph node metastasis as well as differentiated degrees of the tumor $(P=0.003,0.017,0.000 ; P=0.001,0.008,0.003)$ instead of the age, tumor size and clinical staging $(P>0.05)$. Conclusions: Epithelial-mesenchymal transition (EMT) tends to appear in poorly-differentiated CSCC tissue, and the up-regulation of vimentin expression is accompanied by high expression of $\mathrm{Ki}-67$, suggesting that invasion and metastasis readily occur in these tumor cells.
\end{abstract}

Keywords: Cervical squamous cell carcinoma - vimentin - Ki-66 - EMT

Asian Pac J Cancer Prev, 16 (10), 4271-4275

\section{Introduction}

Cervical cancer is one of the most common malignant tumors in the female reproductive system, and its incidence is only secondary to breast cancer in developing countries. The occurrence of squamous cell carcinoma which is the most common histological type of cervical cancer is a dynamic and continuous process from cervical intraepithelial neoplasia (CIN) to invasive carcinoma (Muñoz et al., 2014). With screening of cervical cancer developing extensively in recent years, the early diagnosis and treatment of cervical cancer and precancerous lesions have been improved conspicuously, but infiltration, metastasis and poor differentiation are still important reasons for postoperative recurrence and deaths of patients with cervical cancer (Liu et al., 2014; Qureshi et al., 2014). Therefore, to investigate the infiltrative and metastatic mechanisms of cervical cancer is of great importance for its treatment and prognosis.

Epithelial-mesenchymal transition (EMT) is a key step in the process of cancer metastasis (Beuran et al., 2015). Increase of Vimentin expression and decrease of E-cadherin expression are the major indexes for EMT incidence. The study has confirmed that up-regulation of Vimentin expression in various epithelial tumors is intimately associated with the tumor migration, invasion and metastasis (Satelli et al., 2011). Differentiation of tumor cells is mutually antagonistic to their proliferation, namely the more active the cell proliferation is, and the worse the differentiated degrees are. Ki-67, a sort of protein related to cell proliferation, can reflect the proliferative capability of tumor cells, and its expression is associated with the malignant degrees of tumor and the patient's poor diagnosis (Chen et al., 2015). In this study, the expression of Vimentin and Ki-67 proteins in cervical squamous cell carcinoma (CSCC) was analyzed, and their correlation with the patients' clinicopathological features was also investigated so as to provide more clinical evidences for the biological behaviors and prognostic assessment of cervical cancer.

\section{Materials and Methods}

\section{Sample source}

Fifty-seven CSCC samples archived in Department of Pathology and confirmed by two pathologists in The

${ }^{1}$ Department of Gynecology, ${ }^{2}$ Department of Obstetrics and Gynecology, The First Affiliated Hospital of Wenzhou Medical University, Wenzhou, China*For correspondence: baokang1124@sina.cn 
Jian-Qin Yu et al

First Affiliated Hospital of Wenzhou Medical University were selected from March 2012 to March 2014. All cases had complete clinicopathological files. According to International Federation of Gynecology and Obstetrics (FIGO) staging criteria, there were 34 cases at phase Ib and 23 at phase IIa. The patients with poor, moderate and high differentiation were 28,19 and 10 cases, respectively. Besides, all the enrolled samples were approved by Ethics Committee of The First Affiliated Hospital of Wenzhou Medical University, and the patients or their relatives signed informed consent form.

\section{Reagent}

Mouse anti-human Vimentin monoclonal antibody was purchased from Wuhan Boster Biotech Co., Ltd, and mouse anti-human Ki-67 monoclonal antibody from American ZYMED Corporation. Universal secondary antibody kits were provided by Beijing Zhongshan Golden Bridge Biotech Co., Ltd.

\section{Immunohistochemical SP method}

All samples were fixed by paraformaldehyde, embedded by paraffin and cut into slices with the thickness of $2 \mu \mathrm{m}$. The expression of Vimentin and $\mathrm{Ki}-67$ proteins in CSCC tissue was detected using immunohistochemical SP method. Specific steps were as follows: First, the sections were given dewaxing and debenzylation to hydration by gradient xylene and alcohol; Second, the antigens were repaired with high pressure in citrate for 2 min. Endogenous catalase was sealed with $3 \%$ hydrogen peroxide for $8 \mathrm{~min}$ after the antigens were cooling to the room temperature; Third, primary antibodies were used for incubation, overnight, and then phosphate buffer solution (PBS) was applied to swash three times; Fourth, DAB and hematoxylin were respectively used for coloration and counterstaining after incubation for $50 \mathrm{~min}$ by secondary antibodies; At last, the neural resin was applied to seal the sections and staining results were observed after hydration and transparency by gradient xylene and alcohol.

\section{Criteria on evaluation}

All sections were observed and judged independently by two pathologists under the condition of unknown clinical data. The positive expression of Vimentin was defined as presence of yellow and brown yellow granules in tumor cytoplasma, while that of $\mathrm{Ki}-67$ as presence of yellow and brown yellow granules in tumor nuclei. The final score of immunohistochemistry was calculated using semi-quantitative method based on staining intensity and the percentage of positive cells. Three high-power visual fields $(\times 400)$ in each section were randomly selected, and 100 CSCC cells in each visual field were also selected again. First of all, the scores were made according to staining intensity, namely colorless, light yellow, brown yellow and sepia were represented by $0,1,2$, and 3 points, respectively. Next, the scores were made again based on the percentage of positive cells, that is $0,1,2,3$ and 4 points were on behalf of the negative, the percentage of positive cells $\leq 10 \%$, being $11 \% \sim 40 \%$, being $41 \% \sim 75 \%$ and $\geq 76 \%$, respectively. The product of two scores was as the final score, namely $0 \sim 1$ point being negative (-),
2 3 points being weakly positive $(+), 4 \sim 7$ points being positive $(++)$ and $\geq 8$ points being strongly positive $(+++)$.

\section{Statistical data analysis}

SPSS 15.0 software package was used for statistical analysis. The enumeration data were compared using $\mathrm{x}^{2}$ test and expressed by percentages. Pearson correlation analysis was carried out for those with normal distribution of Ki-67 and Vimentin expression. The relationships between Ki-67, Vimentin expression and the patients' clinicopathological features were analyzed by $\mathrm{x}^{2}$ test of the four-fold table. All statistical tests were performed in a two-sided way. $\mathrm{P}<0.05$ was considered to be statistically significant.

\section{Results}

Expression of vimentin and $\mathrm{Ki}-67$ in CSCC tissue The positive signals of Vimentin were located in

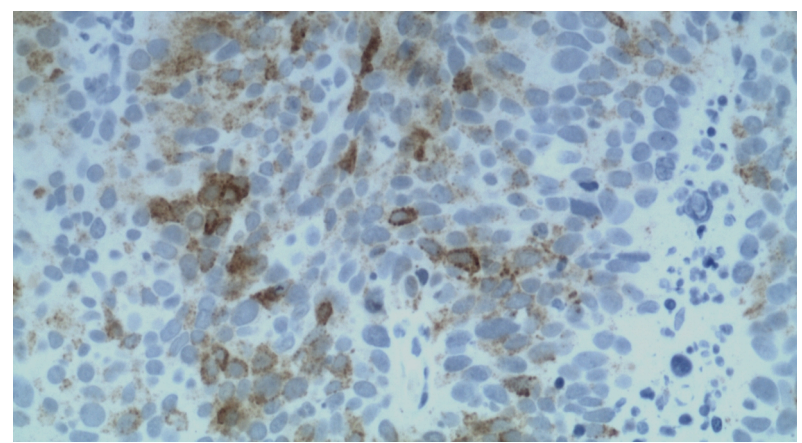

Figure 1. Expression of Vimentin in CSCC Tissue (×400)

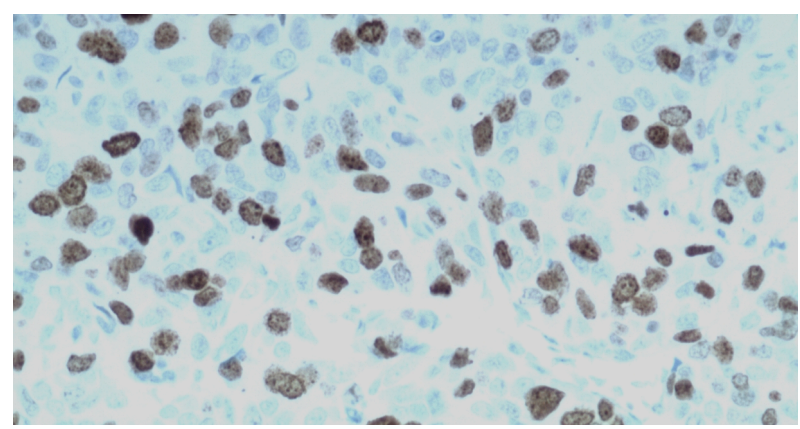

Figure 2. Expression of Ki-67 in CSCC Tissue $(\times 400)$



Figure 3. Correlation between Vimentin and Ki-67 Expression in CSCC Tissue 

Table 1. Relationship between Ki-67 Expression and the Clinicopathological Features of Patients with CSCC $[\mathbf{n}(\%)]$

\begin{tabular}{|c|c|c|c|c|c|c|}
\hline \multicolumn{2}{|c|}{ Clinicopathological features } & \multirow{2}{*}{$\begin{array}{c}\mathrm{n} \\
33\end{array}$} & \multicolumn{2}{|c|}{$\begin{array}{l}\text { Ki-67 Vimentin expression } \\
\text { Weakly positive Strongly positive }\end{array}$} & \multirow{2}{*}{$\begin{array}{c}\chi^{2} \\
0.025\end{array}$} & \multirow{2}{*}{$\begin{array}{c}\mathrm{P} \\
0.875\end{array}$} \\
\hline Age (years old) & $\leq 45$ & & $9(27.3)$ & $24(72.7)$ & & \\
\hline & $>45$ & 24 & $7(29.2)$ & $17(70.8)$ & & \\
\hline \multirow{2}{*}{ Tumor size $(\mathrm{cm})$} & $\leq 4$ & 46 & $13(28.3)$ & $33(71.7)$ & 0.004 & 0.948 \\
\hline & $>4$ & 11 & $3(27.3)$ & $8(72.7)$ & & \\
\hline \multirow[t]{2}{*}{ Local invasion } & Yes & 25 & $2(8.0)$ & $23(92.0)$ & 8.884 & 0.003 \\
\hline & No & 32 & $14(43.8)$ & $18(56.3)$ & & \\
\hline \multirow[t]{2}{*}{ FIGO staging } & $\mathrm{Ib}$ & 34 & $8(23.5)$ & $26(76.5)$ & 0.861 & 0.354 \\
\hline & IIa & 23 & $8(34.8)$ & $15(65.2)$ & & \\
\hline \multirow{2}{*}{ Differentiated degrees } & Poor differentiation & 28 & $1(3.6)$ & $27(96.4)$ & & \\
\hline & Moderate and high differentiation & 29 & $15(51.7)$ & $14(48.3)$ & & \\
\hline \multirow[t]{2}{*}{ Lymph node metastasis } & Yes & 21 & 1(4.8) & $20(95.2)$ & 7.035 & 0.008 \\
\hline & No & 36 & $14(38.9)$ & $22(61.1)$ & & \\
\hline
\end{tabular}

Table 2. Relationship between Vimentin Expression and the Clinicopathological Features of Patients with CSCC [n(\%)]

\begin{tabular}{|c|c|c|c|c|c|c|}
\hline \multicolumn{2}{|c|}{ Clinicopathological features } & \multirow{3}{*}{$\begin{array}{c}\mathrm{n} \\
33\end{array}$} & \multicolumn{2}{|c|}{ Vimentin expression } & \multirow[t]{2}{*}{$\chi^{2}$} & \multirow[t]{2}{*}{$P$} \\
\hline & & & Negative & positive & & \\
\hline \multirow[t]{2}{*}{ Age (years old) } & $\leq 45$ & & $9(27.3)$ & $24(72.7)$ & \multirow[t]{2}{*}{0.311} & \multirow[t]{2}{*}{0.577} \\
\hline & $>45$ & 24 & $5(20.8)$ & $19(79.2)$ & & \\
\hline \multirow[t]{2}{*}{ Tumor size (cm) } & $\leq 4$ & 46 & $13(28.3)$ & $33(71.7)$ & \multirow[t]{2}{*}{1.761} & \multirow[t]{2}{*}{0.185} \\
\hline & $>4$ & 11 & $1(9.1)$ & $10(90.9)$ & & \\
\hline \multirow[t]{2}{*}{ Local invasion } & Yes & 25 & $1(4.0)$ & $24(96.0)$ & \multirow[t]{2}{*}{10.161} & \multirow[t]{2}{*}{0.001} \\
\hline & No & 32 & $13(40.6)$ & $19(59.4)$ & & \\
\hline \multirow[t]{2}{*}{ FIGO staging } & $\mathrm{Ib}$ & 34 & $10(29.4)$ & $24(70.6)$ & \multirow{4}{*}{9.012} & \multirow{4}{*}{0.003} \\
\hline & $\mathrm{IIa}$ & 23 & $4(17.4)$ & $19(82.6)$ & & \\
\hline \multirow[t]{2}{*}{ Differentiated degrees } & Poor differentiation & 28 & $2(7.1)$ & $26(92.9)$ & & \\
\hline & Moderate and high differentiation & 29 & $12(41.4)$ & $17(58.6)$ & & \\
\hline \multirow[t]{2}{*}{ Lymph node metastasis } & Yes & 21 & 1(4.8) & $20(95.2)$ & \multirow[t]{2}{*}{7.035} & \multirow[t]{2}{*}{0.008} \\
\hline & No & 36 & $13(36.1)$ & $23(63.9)$ & & \\
\hline
\end{tabular}

cytoplasma, and it was primarily expressed in some cervical cancer cells and interstitial tissue (Figure 1). Semi-quantitative scoring in this study was only used to calculate the cervical cancer cells. The positive signals of Ki-67 were located in nuclei, mainly manifesting brow yellow or sepia granules (v 2).

Relationship between Ki-67 expression and the clinicopathological features of patients with CSCC

Ki-67 expression was intimately associated with the presence or absence of local invasion and lymph node metastasis as well as differentiated degrees of the tumor $(\mathrm{P}=0.003,0.017,0.000)$ instead of the age, tumor size and clinical staging $(\mathrm{P}>0.05)$ (Table 1$)$.

Relationship between Vimentin expression and the clinicopathological features of patients with CSCC

Vimentin expression was also closely related to the presence or absence of local invasion and lymph node metastasis as well as differentiated degrees of the tumor $(\mathrm{P}=0.001,0.008,0.003)$, but not to the age, tumor size and clinical staging $(\mathrm{P}>0.05)$ (Table 2$)$.

\section{Discussion}

CSCC is one of the most common cervical cancers in female reproductive system, approximately accounting for $80 \%$ of the total (Gan et al., 2014). According to the statistics, the newly-onset patients are about 5 million in the world every year, in which about $50 \%$ mainly focus on the developing countries. In recent years, more and more young women tend to suffer from cervical cancer (Li et al., 2009). There is a long period of precancerous lesions from cervical cells to cancer cells. The survival rate of patients with early cervical cancer comes up to $90 \%$. Hence, early prevention and treatment can effectively reduce the occurrence of cervical cancer and mortality.

Cervical cancer is a sort of polygenic disease, and its occurrence is related to a lot of factors, in which the most pivotal one is continuous infection of high-risk human papilloma virus (HPV) (Gonzalez-Herrera et al., 2015). Geiger et al. found that the phenotype of epithelial cells in normal epithelial cells infected by HPV gradually disappeared, whereas EMT happened in the phenotype of some mesenchymal cells (Geiger et al., 2008). HPV16 E7 protein can promote the transformation of human normal epithelial cells to mesenchymal cells, and its oncogene proteins can accelerate the tumor malignant transformation through induction of EMT (Hellner et al., 2009). In the processes of tumorigenesis and progression, the mutual actions of transcription factors, protein kinases of tumor microenvironment and other regulatory factors can result in abnormal expression of EMT-related markers. For instance, cytoskeletal rearrangement, loss expression of keratin and up-regulated expression of Vimentin can all cause cell-matrix adhesion and change cell phenotypes, 
consequently enhancing cell invasiveness and metastatic capability (Araki et al., 2010). As a type III intermediate filament protein, Vimentin participates in cytoskeletal formation. As a signal factor, it takes part in crucial life activities, including gene transmission and expression as well as intracellular information transmission. Vimentin can make the epithelial cytoskeleton reconstitute via ectopic expression in epithelial tissue. After biological behaviors and morphology change, it changes into fibroblast-like cells which are easier to move so as to transform into mesenchyme, produce EMT and promote tumor invasion and metastasis. In recent years, the studies have displayed that up-regulation of Vimentin expression in cancer tissue is involved in EMT process, and infiltration and metastasis tend to appear in the tumors where EMT occurred (Xiao et al., 2012). The study made by Cheng et al. has also confirmed that Vimentin is not expressed in normal epithelial cells, but is expressed mesenchymal cells and part of ectoderm cells. It can be considered as a specific marker for indentifying the origin of tumor differentiation (Cheng et al., 2012). At present, there are few studies on Vimentin expression in cervical cancer. Therefore, this study detected the expression of Vimentin in CSCC tissue using immunohistochemical method, and the results showed that 43 out of 57 patients with CSCC had positive expression of Vimentin, and the positive rate was $75.4 \%$; Vimentin expression was also closely related to the presence or absence of local invasion and lymph node metastasis as well as differentiated degrees of the tumor, indicating that EMT is present in the process of CSCC progression, and Vimentin is associated with local invasion and metastasis of cervical cancer.

$\mathrm{Ki}-67$, a sort of nucleoprotein related to cell proliferation, was first discovered in Hodgkin's lymphoma L428 cell lines and cloned. It is composed of two polypeptide chains (345 KD and $395 \mathrm{KD}$ ) and has nonhistone characteristics. The expression and localization of Ki-67 as a nuclear antigen in the cell cycle change periodically due to DNA replication. It can accurately reflect the phase of cell proliferation, namely it begins to express in phase $\mathrm{G} 1$ and reduces immediately after mitosis, but not express in phase G0 (Li et al., 2015). As one of the most extensively-applied cell proliferation markers, $\mathrm{Ki}-67$ is regarded as a more reliable marker that can comprehensively reflect the activity of cell proliferation. It can quantitatively analyze the growth fraction and is of great importance in the tumor grading, proliferative degrees and prognosis (Li et al., 2015; Kontzoglou et al., 2013). A lot of studies displayed that Ki-67 was highly expressed in a variety of gynecological malignant tumors, such as ovarian cancer, endometrial carcinoma and breast cancer, and was related to the tumor occurrence, progression, invasion and metastasis intimately (Kilickap et al., 2014; Kucukgoz et al., 2014; Shevra et al., 2015). The research results in this study revealed that 57 patients with CSCC all had positive expression of $\mathrm{Ki}-67$, and the positive rate came up to $100.0 \%$; Ki-67 was positively correlated with Vimentin expression. Additionally, Ki-67 expression was intimately associated with the presence or absence of local invasion and lymph node metastasis as well as differentiated degrees of the tumor. All these results suggest that the differentiated degrees of tumor are related to occurrence of EMT, and EMT tends to appear in the poorly-differentiated tumor tissue.

To sum up, EMT is present in the process of CSCC progression and tends to appear in the poorly-differentiated tumor tissue. Up-regulation of Vimentin expression in CSCC tissue is accompanied by high expression of $\mathrm{Ki}-67$, suggesting that invasion and metastasis are easy to occur in these tumor cells. Combined application of Vimentin and $\mathrm{Ki}-67$ in the postoperative pathological diagnosis is of great importance for judging the biological behaviors of cervical cancer and patients' prognosis.

\section{References}

Araki S, Eitel JA, Batuello CN, et al (2010). TGF-beta1-induced expression of human Mdm2 correlates with late-stage metastatic breast cancer. J Clin Invest, 120, 290-302.

Beuran M, Negoi I, Paun S, et al (2015). The epithelial to mesenchymal transition in pancreatic cancer: A systematic review. Pancreatology, [Epub ahead of print].

Chen WJ, He DS, Tang RX, et al (2015). Ki-67 is a valuable prognostic factor in gliomas: evidence from a systematic review and meta-analysis. Asian Pac J Cancer Prev, 16, 411-20.

Cheng Y, Zhou Y, Jiang W, et al (2012). Significance of E-cadherin, $\beta$-catenin, and vimentin expression as postoperative prognosis indicators in cervical squamous cell carcinoma. Hum Pathol, 43, 1213-20.

Gan LL, Zhang H, Guo JH, et al (2014). Prevalence of human papillomavirus infection in oral squamous cell carcinoma: a case-control study in Wuhan, China. Asian Pac J Cancer Prev, 15, 5861-5.

Geiger T, Sabanay H, Kravchenko-Balasha N, et al (2008). Anomalous features of EMT during keratinocyte transformation. PLoS One, 3, 1574.

Gonzalez-Herrera A, Salgado-Bernabe M, Velazquez-Velazquez $C$, et al (2015). Increased expression of HOXB2 and HOXB13 proteins is associated with HPV infection and cervical cancer progression. Asian Pac J Cancer Prev, 16, 1349-53.

Hellner K, Mar J, Fang F, et al (2009). HPV16 E7 oncogene expression in normal human epithelial cells causes molecular changes indicative of anepithelial to mesenchymal transition. Virology, 391, 57-63.

Kilickap S, Kaya Y, Yucel B, et al (2014). Higher Ki67 expression is associates with unfavorable prognostic factors and shorter survival in breast cancer. Asian Pac J Cancer Prev, 15, 1381-5.

Kontzoglou K, Palla V, Karaolanis G, et al (2013). Correlation between Ki67 and breast cancer prognosis. Oncology, 84, 219-25.

Kucukgoz Gulec U, Gumurdulu D, Guzel AB, et al (2014). Prognostic importance of survivin, Ki-67, and topoisomerase II $\alpha$ in ovarian carcinoma. Arch Gynecol Obstet, 289, 393-8.

$\mathrm{LiH}$, Diao YT, Ma JX, et al (2009). Time trends on the prevalence of cancer during 1970-2005 in Shandong province. Chinese Journal of Epidemiology, 30, 592-5.

Li LT, Jiang G, Chen Q, et al (2015). Ki67 is a promising molecular target in the diagnosis of cancer. Mol Med Rep, 11, 1566-72.

Li PL, Tan HZ (2015). Expression of PPAR $\gamma$, p27 and Ki67 in cervical cancer and its clinical significance. $J$ Int Transl Med, 3, 513-9.

Liu YQ, Li HF, Han JJ, et al (2014). CD44v3 and VEGF-C expression and its relationship with lymph node metastasis 

in squamous cell carcinomas of the uterine cervix. Asian Pac J Cancer Prev, 15, 5049-53.

Muñoz N, Bravo LE (2014). Epidemiology of cervical cancer in Colombia. Salud Publica Mex, 56, 431-9.

Qureshi R, Arora H, Rizvi MA (2014). EMT in cervical cancer: its role in tumour progression and response to therapy. Cancer Lett, 356, 321-31.

Satelli A, Li S (2011). Vimentin in cancer and its potential as a molecular target for cancer therapy. Cell Mol Life Sci, 68, 3033-46.

Shevra CR, Ghosh A, Kumar M (2015). Cyclin D1 and Ki67 expression in normal, hyperplastic and neoplastic endometrium. J Postgrad Med, 61, 15-20.

Xiao S, Liu L, Lu X, et al (2012). The prognostic significance of bromodomain PHD-finger transcription factor in colorectal carcinoma and association with vimentin and E-cadherin. $J$ Cancer Res Clin Oncol, 2015, [Epub ahead of print]. 